International Mathematical Forum, Vol. 9, 2014, no. 14, 651 - 659

HIKARI Ltd, www.m-hikari.com

http://dx.doi.org/10.12988/imf.2014.312232

\title{
On Geometric Hyper-Structures ${ }^{1}$
}

\section{Mashhour I.M. Al Ali Bani-Ata, Fethi Bin Muhammad Belgacem and Abdulhamid Al-ibrahim}

\author{
Department of Mathematics, PAAET, Shamieh, Kuwait
}

Copyright (c) 2014 Mashhour I.M. Al Ali Bani-Ata et al. This is an open access article distributed under the Creative Commons Attribution License, which permits unrestricted use, distribution, and reproduction in any medium, provided the original work is properly cited.

\begin{abstract}
The aim of this paper is to introduce the notion of hyper-semifields, hyper-spread sets and hyper-spreads on one hand and to investigate the relation among these structures.
\end{abstract}

\section{Introduction}

We recall some hyper-structures theory. A hyper-groupoid $(H ; \otimes)$ is the set $H$ endowed with a binary multi-valued operation (hyper-operation) i.e a function $\otimes$ from $H \times H$ to $\rho^{*}(H)$ the non empty set of subsets of $H$. A quasihyper-group is a hyper-groupoid such that $x \otimes H=H \otimes x=H \quad \forall x \in H$, (the reproduction axiom), where $H \otimes x=\bigcup_{h \in H} h \otimes x$. A semi-hyper-group is a hyper-groupoid $(H, \otimes)$ such that $(x \otimes y) \otimes z=x \otimes(y \otimes z) \quad \forall(x, y, z) \in H^{3}$. A semi-hyper-group $(H, \otimes)$ is a hyper-group (or also multi-group) if $H \otimes x=$ $x \otimes H=H \quad \forall x \in H$, or equivalently if for all $(a, b) \in H^{2}$, there exists $(c, d) \in H^{2}$ such that $b \in c \otimes a, b \in a \otimes d$.

The condition $(x \otimes y) \otimes z=x \otimes(y \otimes z)$ can be rephrased as : $\bigcup_{u \in x \otimes y} u \otimes z=$

\footnotetext{
${ }^{1}$ This project is supported by Research adminstration-Paaet project no. BE-11-15
} 
$\bigcup_{v \in y \otimes z} x \otimes v$. For more information about hyper-structures one may refer to [4], $[2],[1],[3]$ and [5].

\section{Preliminaries ANd EARlier Studies}

Definition 1. If $(H, \otimes)$ and $\left(H^{\prime}, *\right)$ are two hyper-groupoids, the function $\Phi$ : $H \rightarrow H^{\prime}$ is called a good homomorphism if and only if $\Phi(x \otimes y)=\Phi(x) * \Phi(y)$ for all $(x, y) \in H^{2}$.

Definition 2. Let $(G, \circ)$ be a hyper-groupoid. The action of $(G, \circ)$ on a non empty set $A$ is a map $\bullet: G \times A \rightarrow \rho *(A)$ such that for all $\left(g_{1}, g_{2}\right) \in G^{2}, a \in A$ :

(i) $\bigcup_{t \in g_{1} \circ g_{2}} t \bullet a=\bigcup_{s \in g_{2} \bullet a} g_{1} \bullet s$

(ii) There exists $e \in G$ such that $a \in e \bullet a$.

Remark 1. Let $A^{\rho(A)}$ be the set of all functions from $A$ to $\rho(A)$, endowed with the composition operation $\circ$, then $\Phi: G \rightarrow G^{\rho(A)}$ defined by $\Phi(g)(a)=g \bullet a$ is a homomorphism.

Proof. $\left(\Phi\left(g_{1} \circ g_{2}\right)\right)(a)=\bigcup_{t \in g_{1} \circ g_{2}} t \bullet a$. From the above definition one obtains $\bigcup_{t \in g_{1} \circ g_{2}} t \bullet a \bigcup_{s \in g_{2} \bullet a} g_{1} \bullet s=g_{1} \bullet\left(g_{2} \bullet a\right)=\Phi\left(g_{1}\right)\left(\Phi\left(g_{2}\right)\right)(a)=\left(\Phi\left(g_{1}\right) \circ \Phi\left(g_{2}\right)\right)(a)$.

The homomorphism $\Phi: G \rightarrow A^{\rho(A)}$ is called a hyper-representation associated with the hyper-groupoid action. This process is reversible in the sense that if $\Phi: G \rightarrow A^{\rho(A)}$ is any good homomorphism, then the map from $G \times A \rightarrow \rho(A)$ defined by $g \bullet a=\Phi(g)(a)$ satisfies the properties of hyper-groupoid action of $G$ on $A$.

\section{HyPER-GEOMETRIC NOTATIONS}

Definition 3. A hyper-vector space is a quadraplet $(V,+, \circ, K)$, where $(V,+)$ is abelian group, $K$ is a field and $\circ$ is a mapping (product times a scalar in $K)$. $\circ: K \times V \rightarrow \rho^{*}(V)$ where $\rho^{*}(V)$ is the non empty subsets of $V$, such that the following conditions hold:

(i) For all $a \in K$ and for all $x, y \in V$, then $a \circ(x+y) \subseteq a \circ x+a \circ y$.

(ii) For all $a, b \in K$ and for all $x \in V$, then $(a+b) \circ x \subseteq a \circ x+b \circ x$.

(iii) For all $a, b \in K$ and for all $x \in V$, then $a \circ(b \circ x)=(a \cdot b) \circ x$.

(iv) For all $a, b \in K$ and for all $x \in V$, then $a \circ(-x)=(-a) \circ x=-(a \circ x)$. 
(v) For all $x \in V$, then $x \in 1 \circ x$.

In the right hand side of (i), the sum is meant in the sense of Frobenius, that is

$$
a \circ x+a \circ y=\{w+u \mid w \in a \circ x, u \in a \circ x\} .
$$

Definition 4. A hyper-algebra $V$ over a field $K$ is a triple $(V, \odot, K)$ where $(V,+, \circ, K)$ is a hyper-vector space over $K$, and $\odot$ is a hyper-operation $\odot$ : $V \times V \rightarrow \rho^{*}(V)$ such that the following conditions hold:

(i) There is no element $e \in V$, such that $x \in x \odot e \cap e \odot x$ for all $x \in V$, i.e $e$ is a right unit element of $V$.

(ii) $x \odot(y+z)=x \odot y+x \odot z$ for all $x, y, z \in V$ and $(x+y) \odot z=x \odot z+y \odot z$ for all $x, y, z \in V$, where the right hand side of (ii) are meant the Frobenius.

(iii) If $t \in K$, then $(t \circ a) \odot b=t \circ(a \odot b)=a \odot(t \circ b)$ for all $t \in K$ and for all $a, b \in V$, where $(t \circ a) \odot b=\bigcup_{w \in t \circ a} w \odot b, t \circ(a \odot b)=\bigcup_{u \in a \odot b} t \circ u$ and $a \odot(t \circ b)=\bigcup_{s \in t \circ a} a \odot s$.

Definition 5. A hyper-semifield over a field $K$, is a hyper-algebra $(V, \odot, K)$ such that $V$ has no zero divisors, i.e if $0 \neq a \in V$ and $b \in V$ such that $0 \in a \odot b$, then $0 \in b \odot e$.

Definition 6. Let $(V, \odot, K)$ and $(W, \oplus, F)$ be two hyper-semifields over the fields $K$ and $F$ respectively, then a good hyper-homomorphism $\sigma: V \rightarrow W$ is a mapping such that $\sigma(x \odot y)=\sigma(x) \oplus \sigma(y)$ for all $x, y \in V$.

Definition 7. If $(V,+, \circ, K)$ is a hyper-vector space over $K$, then a hyperlinear transformation $\delta: V \rightarrow \rho^{*}(V)$ is defined by:

(i) $\delta(v+w)=\delta(v)+\delta(w)$ for all $u, w \in V$.

(ii) $\delta(t \circ a)=t \circ \delta(a)$ for all $t \in K$ and $a \in V$.

The set of all hyper-linear transformations of $V$ is denoted by $H L(V)$. In particular if $(V, \odot, K)$ is a hyper-semifield over $K$, then a hyper-linear transformation $\delta$ of $V$ is called a hyper-faithful-linear transformation if $0 \in$ $\delta(b), b \in V$ then $0 \in e \odot b$, i.e $e$ is the unite element of $(V, \odot, K)$.

Proposition 1. Let $(V, \odot, K)$ be a hyper-semifield over a field $K$. For $a \in V$, let the hyper-mappings $\alpha_{a}: V \rightarrow \rho^{*}(V)$ and $\alpha_{a}^{*}: V \rightarrow \rho^{*}(V)$ defined by $\alpha_{a}(v)=a \odot v$ and $\alpha_{a}^{*}(v)=v \odot$ a respectively, where $v \in V$, are hyper-faithfullinear transformations.

Proof. Let $a \in V, a \neq 0$, then 
1. $\alpha(x+y)=a \odot(x+y)=a \odot x+a \odot y=\alpha_{a}(x)+\alpha_{a}(y)$. (Use (ii) of Definition 4)

2. $\alpha_{a}(t \circ x)=a \odot(t \circ x)=t \circ(a \odot x)=t \circ\left(\alpha_{a}(x)\right)$ for all $t \in K$ and $x \in V$ (use (iii) of Definition 4). If $0 \in \alpha_{a}(b)$, this implies that $0 \in a \odot b$. As $a \neq$, then from the non-zero divisors property, it follows that $0 \in b \odot e$, which means that $\alpha_{a}$ is hyper-faithful linear transformation.

Definition 8. Let $(V,+, \circ, K)$ be a hyper-vector space over $K$, and let $\Sigma=$ $\left\{\alpha_{a} \mid 0 \neq a \in V\right\} \subseteq H L^{*}(V)$ the set of all hyper-faithful-linear transformations of $V$ over $K$ such that:

(i) $t \cdot \alpha_{S} \subseteq \Sigma$ where $\left(t \cdot \alpha_{S}\right)(x)=t \circ\left(\alpha_{S}(x)\right)$ for all $S, x \in V$ and for all $t \in K$.

(ii) If $\alpha_{S}, \alpha_{T} \in \Sigma$, then $\alpha_{S}+\alpha_{T} \in \Sigma$ where $\left(\alpha_{S}+\alpha_{T}\right)(x)=\alpha_{S}(x)+\alpha_{T}(x)$, for all $x \in V$.

(iii) There exists $I \in \Sigma$ such that $x \in I(x)$, for all $x \in V$.

(iv) There is a fixed element $e \in V$ and for all non-zero $x \in V$, there exists a unique $T \in \Sigma$ such that $x \in T(e)$.

(v) For all $t \in K$ and for $a, b \in V$, the following condition must hold:

$$
\bigcup_{z \in t \circ a} \alpha_{z}(b)=\bigcup_{w \in \alpha_{a}(b)} t \circ w=\bigcup_{u \in t \circ b} \alpha_{a}(u)
$$

Then $(\Sigma, \cdot,+)$ is called a hyper-spread set over $K$.

Theorem 1. If $(V, \odot, K)$ is a hyper-semifield over $K$, then the hyper-regular representation $\xi: a \rightarrow \alpha_{a}, 0 \neq a \in V$ affords a hyper-spread set $\Sigma$ over $K$.

Proof. Let $\Sigma=\left\{\alpha_{a} \mid 0 \neq a \in V\right\}$, where $\alpha_{a}: V \rightarrow \rho^{*}(V)$ defined by $\alpha_{a}(x)=$ $a \odot x$ is a hyper-faithful-linear transformation, by Proposition 1

(i) If $\alpha_{a}, \alpha_{b} \in \Sigma$, then $\left(\alpha_{a}+\alpha_{b}\right)(x)=\alpha_{a}(x)+\alpha_{b}(x)=a \odot x+b \odot x=$ $(a+b) \odot x=\alpha_{a+b}(x)$. This implies that $\alpha_{a}+\alpha_{b} \in \Sigma$.

(ii) $\left(t \cdot \alpha_{a}\right)(x)=t \circ\left(\alpha_{a}(x)\right)=t \circ(a \odot x)=(t \circ a) \odot x=\alpha_{t \circ a}(x)=\alpha_{w}(x)$, where $w \in t \circ a, t \in K$. This implies that $t \cdot \alpha_{a} \subseteq \Sigma$, for all $t \in K$.

(iii) $I=\alpha_{e} \in \Sigma$ for: $\alpha_{e}(x)=e \odot x$ and as $x \in e \odot x$ for all $x \in V$, then it follows that $\alpha_{e}(x)=I(x)$.

(iv) As $x \in x \odot e$ for all $x \in V$, it follows that there exists a unique $\alpha_{x}$ such that $x \in \alpha_{x}(e)$. Setting $\alpha_{x}=T$, thus, there is a unique $T \in \Sigma$ such that $x \in T(e), x \in V$.

Hence the claim follows.

Theorem 2. Let $\Sigma$ be a hyper-spread set over a field $K$, then $\Sigma$ affords a hyper-semifield over $K$. 
Proof. As $\Sigma \subseteq H L(V)$ is a hyper-spread set, then pick some vector $0 \neq e \in V$. By ((iv) of Definition 8), and for any $x \in V$, there exists a unique $T \in \Sigma$ (can be called $\left.T_{x}\right)$ such that $x \in T_{x}(e)$. Hence we can define a hyper-multiplication operation $\odot$ on $V$ as follows: $x \odot y=T_{x}(y)$, where $x \in T_{x}(e)$ for all $x, y \in V$. This turns $V$ into a hyper-semifield over $K$ for: $x \odot(y+z)=T_{x}(y+z)=$ $T_{x}(y)+T_{x}(z)=x \odot y+x \odot z$.

As $x+y \in V$, then there exists a unique $T_{x+y} \in \Sigma$ such that $(x+y) \in$ $(x+y) \odot e=T_{(x+y)}(e)$.

Arguing similarly $x+y \in x \odot e+y \odot e=T_{x}(e)+T_{y}(e)$. From the uniqueness property of $T_{x+y}, T_{x}$ and $T_{y}$, it follows that $T_{x}+T_{y}=T_{x+y}$ and hence

$$
\begin{aligned}
\left(T_{x+y}\right)(z) & =(x+y) \odot z=\left(T_{x}+T_{y}\right)(z) \\
& =T_{x}(z)+T_{y}(z)=x \odot z+y \odot z, \quad \forall x, y, z \in V
\end{aligned}
$$

Similarly one can show that $x \odot(y+z)=x \odot y+x \odot z$ for all $x, y, z \in V$.

From ((iv) Definition 8), there is $e \in V$ such that there exists a unique $T \in \Sigma$ such that $x \in T(e)=T_{x}(e)=x \odot e$. This implies that this $e$ is the unit element of $V$.

From ((v) Definition 8), it follows that:

$$
\begin{aligned}
(t \circ a) \odot x & =T_{t \circ a}(x)=\bigcup_{z \in t \circ a} T_{z}(x)=\bigcup_{w \in \alpha_{a}} t \cdot w \\
& =t \circ\left(\alpha_{a}(b)\right)=\bigcup_{u \in t \circ b} T_{a}(u)=T_{a}(t \circ b)
\end{aligned}
$$

for all $t \in K$ and for all $a, b \in V$.

The property of has no zero-divisors is an immediate consequence from the faithful condition of elements of $\Sigma$.

This completes the proof of the theorem.

Definition 9. Let $(V,+, \circ, K)$ be a hyper-vector space over $K$. Then $H \subseteq V$ is a hyper-subspace of $V$ if $(H,+, \circ, K)$ is a hyper-vector space over $K$.

Definition 10. Let $(V,+, \circ, K)$ be a hyper-vector space, a hyper-spread over $K$ is a collection $\mathfrak{K}$ of hyper-subspaces of $V$ such that:

1. If $X, Y \in \mathfrak{K}$ and $X \neq Y$, then $0 \in X \cap Y$.

2. $\bigcup_{X \in \mathfrak{K}} X=V$. The elements of $\mathfrak{K}$ are called components.

Lemma 1. Let $(V,+, \circ, K)$ be a hyper-vector space, then $A=V \times V$ can be turned into a hyper-vector space as follows:

Define the hyper-operation $\oplus: V \times V \rightarrow \rho^{*}(V)$

$$
(a, b) \oplus(c, d)=(a \oplus c, b+d), \quad \forall a, b, c, d \in V,
$$


and define the hyper-operation $\hat{o}: K \times(V \times V) \rightarrow \rho^{*}(V)$

$$
t \hat{\circ}(a, b)=(t \circ a, t \circ b), \forall a, b \in V, t \in K .
$$

The proof of $(V \times V, \oplus, \hat{o}, K)$ is a hyper-vector space is easy and can be omitted.

Lemma 2. Let $(V, \odot, K)$ be a hyper-semifield, then the subsets $L_{\infty}=\{(x \oplus$ $e, 0) \mid x \in V\}$ and $R_{\infty}=\{(0, y \oplus e) \mid y \in V\}$ of $(V \times V, \oplus, \hat{o}, K)$ are hypersemifields.

Proof. Define the hyper-operation $\hat{\odot}: L_{\infty} \times L_{\infty} \rightarrow \rho^{*}\left(L_{\infty}\right)$ by

$$
(x \odot e, 0) \hat{\odot}(y \odot e, 0)=((x \odot y) \odot e, 0), \forall x, y \in V,
$$

where $\rho^{*}\left(L_{\infty}\right)$ is the set of non-empty subsets of $L_{\infty}$.

Also, the hyper-operations $\hat{o}, \oplus$ on $K \times L_{\infty}$ and $L_{\infty} \times L_{\infty}$ respectively by: tô $(x \odot e, 0)=((t \circ x) \odot e, 0)$ and $(x \odot e, 0) \oplus(y \odot, e, 0)=((x+y) \odot e, 0)$ for all $x, y \in V$ and for all $t \in K$.

(i) The hyper-operation $\hat{\odot}$ is distributive with respect to $\oplus$ in $L_{\infty} \times L_{\infty}$ for

$$
\begin{aligned}
(x \odot e, 0) \hat{\odot}((y \odot e), 0) \oplus(z \odot e, 0)= & (x \odot e, 0) \hat{\odot}((y \oplus z) \odot e, 0) \\
= & ((x \odot(y \oplus z)) \odot e, 0) \\
= & ((x \odot y) \oplus(x \odot z)) \odot e, 0) \\
= & (x \odot y) \odot e, 0) \oplus((x \odot z) \odot e, 0) \\
= & (x \odot e, 0) \odot(y \odot e, 0) \\
& \oplus(x \odot e, 0) \hat{\odot}(z \odot e, 0)
\end{aligned}
$$

Also, one can show that:

$(x \odot e, 0) \oplus((y \odot e), 0) \odot(z \odot e, 0)=(x \odot e, 0) \odot(z \odot e, 0) \oplus(y \odot e, 0) \odot(z \odot e, 0)$

(ii) If $t \in K$, then

$$
\begin{aligned}
& t \hat{o}((x \odot e, 0) \hat{\odot}((y \odot e), 0)=t \hat{o}((x \odot y) \odot e, 0) \\
& =((t \circ(x \odot y) \odot e, 0) \\
& =((x \odot((t \circ y) \odot e, 0) \\
& =(x \odot e) \hat{\odot}((t \circ y) \odot e, 0) \\
& =(x \odot e, 0) \odot((t \circ y) \odot e, 0) \\
& =(x \odot e, 0) \odot(t \hat{o}(y \odot e), 0)
\end{aligned}
$$

Also, as

$$
\begin{aligned}
t \hat{o}((x \odot y) \odot e, 0) & =t \circ((x \odot y) \odot e, 0) \\
& =(t \circ(x \odot y) \odot e, 0) \\
& =(((t \circ x) \odot y) \odot e, 0) \\
& =((t \circ x) \odot e, 0) \hat{\odot}(y \odot e, 0) \\
& =(t \hat{o}(x \odot e, 0) \odot(y \odot e), 0)
\end{aligned}
$$


(iii) $(e \odot e, 0)$ is the unit element of $L_{\infty} \times L_{\infty}$, for $(x \odot e, 0) \odot(e \dot{e}, 0)=((x \odot$ $e) \odot e, 0)$ for all $(x \odot e, 0)$ in $L_{\infty} \times L_{\infty}$, and as $x \in x \odot e \cap e \odot x$ for all $x \in V$, then $x \odot e \subseteq \bigcup_{w \in x \odot e} w \odot e=(x \odot e) \odot e$. This implies that $(x \odot e, 0) \subseteq((x \odot e) \odot e, 0)=(x \odot e, 0) \odot(e \odot e, 0)$.

Following a similar argument one can show that $(x \odot e, 0) \subseteq(e \odot e) \odot(x \odot$ $e, 0)$

(iv) Assume that $0 \in(x \odot e, 0) \odot(y \odot e, 0)$, where $0 \notin(x \odot e, 0)$, then $0 \in(x \odot$ $y) \odot e, 0)$. This implies that $0 \in(x \odot y) \odot e$. Since $0 \notin e \odot e, 0 \in(x \odot y)$. But $0 \notin x \cdot e$, this implies that $0 \in y \odot e$ and thus $0 \in(y \odot e, 0)$. This completes the proof that $L_{\infty}$ is a hyper-semifield. A similar argument can be followed to prove that $R_{\infty}$ is a hyper-semifield.

Lemma 3. Let $(V, \odot, \hat{o}, \oplus, K)$ be a hyper-semifield, then $\left(L_{\infty} \hat{\times} R_{\infty}, \hat{\oplus}, K\right)$ is a hyper-vector space, where $L_{\infty} \hat{\times} R_{\infty}=\{(\overline{x \odot e}, \overline{y \odot e}) \mid x, y \in V\}$, $(\overline{x \odot e}, \overline{y \odot e}) \hat{\oplus}(\overline{a \odot e}, \overline{b \odot e})=(((x \odot e), 0) \oplus(a \odot e, 0),(0,(y \odot e) \oplus(0, b \odot e)))$ and

$$
t \bar{\circ}(\overline{x \odot e}, \overline{y \odot b})=((t \hat{o}(x \odot e), 0), t \hat{o}(0,(y \odot e)))
$$

for all $x, y, a, b \in V, \overline{x \odot e}=(x \odot e, 0)$.

Proof. The proof is an immediate consequence of Lemma 1 and Lemma 2.

Theorem 3. Let $(V, \odot, K)$ be a hyper-semifield, then $\left(L_{\infty} \hat{\times} R_{\infty}, \wedge, K\right)$ is a hyper-semifield, where

$$
(\overline{x \odot e}, \overline{y \odot e}) \wedge(\overline{a \odot e}, \overline{b \odot e})=(((x \odot e), 0) \hat{\odot}(a \odot e, 0),(0, y \odot e) \hat{\odot}(0, b \odot e))
$$

, where $\overline{x \odot e}=(x \odot e, 0) \forall x \in V$.

$$
\begin{aligned}
& \text { Proof. } \quad \text { 1. }(\overline{x \odot y}, \overline{y \odot e}) \wedge((\overline{a \odot e}, \overline{b \odot e}) \hat{\oplus}(\overline{c \odot e}, \overline{d \odot e}))= \\
& \quad(x \odot e, y \odot e) \wedge((a \odot e, 0) \oplus(c \odot e, 0),(0, b \odot e) \oplus(0, d \odot e))= \\
& \quad(x \odot e, y \odot e) \wedge(a+c) \odot e,(b+d) \odot e)= \\
& \quad(((x \odot e, 0) \hat{\odot}(a \odot e, 0) \oplus(x \odot e, 0) \hat{\odot}(c \odot e, 0),(0, y \odot e) \hat{\odot}(0, b \odot e) \\
& \quad(0, y \odot e) \odot(0, d \odot e)) \\
& =(\overline{x \odot e}, \overline{y \odot e}) \wedge(\overline{a \odot e}, c \odot e) \hat{\oplus}(\overline{x \odot e}, \overline{y \odot e}) \wedge(\overline{c \odot e}, \overline{d \odot e})
\end{aligned}
$$

Arguing in a similar manner one can show that $((\overline{x \odot e}, \overline{y \odot e}) \hat{\oplus}(\overline{a \odot e}, \overline{b \odot e})) \wedge(\overline{c \odot e}, \overline{d \odot e})=$ $((\overline{x \odot e}, \overline{y \odot e}) \wedge(\overline{c \odot e}, \overline{d \odot e}) \hat{\oplus}(\overline{a \odot e}, \overline{b \odot e}) \wedge(\overline{c \odot e}, \overline{d \odot e})$

From this it follows that $\wedge$ is distributive with respect to $\hat{\oplus}$. 
2. $t \bar{\circ}((\overline{x \odot e}, \overline{y \odot e}) \wedge(\overline{a \odot e}, \overline{c \odot e}))=$ $(t \hat{o}((x \odot e, 0) \hat{\odot}(a \odot e, 0)), t \hat{o}((0, y \odot e) \hat{\odot}(0, b \odot e)))$.

Then (2) follows from ((ii) of Lemma 2).

3. $(\overline{e \odot e}, \overline{e \odot e})$ is the unit element of $L_{\infty} \hat{\times} R_{\infty}$. The proof directly follows from ((iii) of Lemma 2).

4. Assume that $0 \in((\overline{x \odot e}, \overline{y \odot e}) \wedge(\overline{a \odot e}, \overline{b \odot e}))$ such that $0 \notin((x \odot$ $e, 0) \hat{\odot}(a \odot e, 0),(0, y \odot e) \hat{\odot}(0, b \odot e))$. From $(($ iv $)$ of Lemma 2$), 0 \in(a \odot e, 0)$ and $0 \in(0, b \odot e)$ which means that $0 \in(a \odot e, 0) \odot(0, b \odot e)$ and hence $0 \in(\overline{a \odot e}, \overline{b \odot e})$.

Remark 2. Let $(V, \odot, K)$ be a hyper-semifield, then the good hyper-homomorphism $\sigma: V \rightarrow \rho^{*}(V)$, keeps $L_{\infty} \hat{\times} R_{\infty}$ invariant if $e^{\sigma}=\{e\}$.

Proof. Let $(\overline{x \odot e}, \overline{y \odot e}) \in L_{\infty} \hat{\times} R_{\infty}$ then $(\overline{x \odot e}, \overline{y \odot e})^{\sigma}=\left((\overline{x \odot e})^{\sigma},(\overline{y \odot e})^{\sigma}\right)=\left(\left((x \odot e)^{\sigma}, 0\right),\left(0,(y \odot e)^{\sigma}\right)\right)$ $\left(\left(x^{\sigma} \odot y^{\sigma}\right), 0\right),\left(0,\left(y^{\sigma} \odot e^{\sigma}\right)\right)=\left((z \odot e, 0),(0,(w \odot e))=(\overline{z \odot e}, \overline{w \odot e}) \in L_{\infty} \hat{\times} R_{\infty}\right.$, where $z, w \in V$.

Definition 11. Let $(V, \odot, K)$ be a hyper-semifield. A left hyper-nucleus $L_{V}$ of $V$ is defined by

$$
L_{V}=\{a \in V \mid a \odot(x \odot y)=(a \odot x) \odot y, \quad \forall x, y \in V\}
$$

Theorem 4. $K \circ e \subseteq L_{V}$ if and only if $e \in L_{V}$.

Proof. Let $e \in L_{V}$ and consider

$(t \circ e) \odot(x \odot y)=t \circ(e \odot(x \odot y))=t \circ((e \odot x) \odot y)=(t \circ(e \odot x)) \odot y=((t \circ e) \odot x) \odot y$ (use (iii) of Definition 4), where $t \in K, \quad x, y \in V$.

From this it follows that $K \circ e \subseteq L_{V}$.

Conversely: Let $K \circ e \subseteq L_{V}$, this implies that

$$
(t \circ e) \odot(x \odot y)=((t \circ e) \odot x) \odot y)
$$

for all $t \in K$ and all $x, y \in V$. From this one has:

$$
t \circ(e \odot(x \odot y))=(t \circ(e \odot x)) \odot y=t \circ(e \odot x) \odot y
$$

which means that

$$
\bigcup_{w \in e \odot(x \odot y)} t \circ w=\bigcup_{z \in(e \odot x) \odot y} t \circ z
$$

Hence $e \in L_{V}$. 


\section{ACKNOWLEDGMENT}

The authors thank are due to Paaet for the financial support, project no. BE-11-15 and to ICTP for offering library facilities.

\section{REFERENCES}

1. M. Ibrahim Mohammad, On hypergroupoid actions, Riv. Mat. Univ. Parma (6) 4(2001), 245-249.

2. P. Corsini and J. Mittas, New topics in hypergroup theory, Multi-Valued log. 2 (1997), 273-285.

3. P. Corsini, Hypergraphs and hypergroups, Algebra Universalis, 35, (1996), 548-555.

4. P. Corsini, Prolegomena of hypergroup theory, Aviani Editori, Tricesimo, 1993.

5. P. Dembowki, Finite Geometries Springer Verlag, Heidelberg 1968.

\section{Received: December 1, 2014}

